\title{
Erratum to: Thermophilic Hydrogen Production from Renewable Resources: Current Status and Future Perspectives
}

Subramanian Mohan Raj • Suvarna Talluri •

Lew P. Christopher

Published online: 18 April 2012

(C) Springer Science+Business Media, LLC 2012

Erratum to: Bioenerg. Res.

DOI 10.1007/s12155-012-9184-4

The units for $\mathrm{H}_{2}$ yield in Table 1 were printed incorrectly as "mmol/L/h" instead of " $\mathrm{mol} / \mathrm{mol}$ hexose".

The correct version is shown below.

The online version of the original article can be found at $\mathrm{http}: / / \mathrm{dx}$.doi. org/10.1007/s12155-012-9184-4.

S. M. Raj $\cdot$ S. Talluri $\cdot$ L. P. Christopher

Center for Bioprocessing Research and Development,

South Dakota School of Mines and Technology,

Rapid City 57701 SD, USA

L. P. Christopher $(\bowtie)$

Department of Chemical and Biological Engineering,

South Dakota School of Mines and Technology,

Rapid City 57701 SD, USA

e-mail: lew.christopher@sdsmt.edu 
Table 1 Comparison of $\mathrm{H}_{2}$ production capabilities of thermophilic bacteria on different carbon feedstocks

\begin{tabular}{|c|c|c|c|c|c|c|}
\hline Organism & Temp $\left({ }^{\circ} \mathrm{C}\right)$ & Substrate & $\begin{array}{l}\text { Major } \\
\text { metabolites }\end{array}$ & $\begin{array}{l}\mathrm{H}_{2} \text { yield } \\
(\mathrm{mol} / \mathrm{mol} \text { hexose })\end{array}$ & $\begin{array}{l}\text { Vol } \mathrm{H}_{2} \text { productivity } \\
(\mathrm{mmol} / \mathrm{L} / \mathrm{h})\end{array}$ & References \\
\hline Thermotoga maritama DSM 3109 & 80 & Glucose & Acetate & 4.0 & 0.58 & [81] \\
\hline \multirow[t]{4}{*}{ Thermotoga neapolitana DSM 4359} & 85 & Glucose & Acetate and lactate & 3.8 & 0.80 & [61] \\
\hline & 77 & Glucose & Acetate and lactate & 4.1 & 0.86 & [61] \\
\hline & 70 & Glucose & Acetate and lactate & 3.5 & 0.47 & [61] \\
\hline & 65 & Glucose & Acetate and lactate & 3.3 & 0.27 & [61] \\
\hline $\begin{array}{l}\text { Caldicellulosiruptor owensensis } \\
\text { DSM } 13100\end{array}$ & 70 & Glucose & Acetate and lactate & 3.8 & 15.00 & [119] \\
\hline Thermotoga elfii DSM 9442 & 65 & Glucose & Acetate & 3.3 & 4.50 & {$[108]$} \\
\hline \multirow{3}{*}{$\begin{array}{l}\text { Caldicellulosiruptor saccharolyticus } \\
\text { DSM } 8903\end{array}$} & 70 & Glucose & Acetate and lactate & 3.6 & 20.00 & [21] \\
\hline & 70 & Glucose & Acetate & 2.5 & 9.211 .3 & {$[38]$} \\
\hline & 70 & Xylose & Acetate and lactate & $2.2^{\mathrm{a}}$ & 9.211 .3 & [38] \\
\hline C. owensensis DSM 13100 & 70 & Xylose & Acetate and lactate & $2.7^{\mathrm{a}}$ & 9.00 & [119] \\
\hline Thermococcus kodakarensis KOD1 & 85 & Starch & Acetate and alanine & 3.3 & 6.70 & [39] \\
\hline T. neapolitana DSM 4359 & 80 & Potato peels & Acetate and lactate & 3.5 & 13.10 & [55] \\
\hline C. saccharolyticus DSM 8903 & 70 & Wheat straw & ND & 3.8 & $1.58^{\mathrm{b}}$ & [33] \\
\hline $\begin{array}{l}\text { Clostridium thermocellum } \\
\text { ATCC } 27405\end{array}$ & 60 & $\alpha$-cellulose & Acetate, lactate, and ethanol & 1.6 & 5.06 & [52] \\
\hline $\begin{array}{l}\text { C. thermocellum JN4 \& } \\
\text { Thermoanaerobacterium } \\
\text { thermosaccharolyticum GD17 }\end{array}$ & 60 & $\alpha$-cellulose & Butyrate & 1.8 & 55 & [49] \\
\hline \multirow[t]{5}{*}{ C. saccharolyticus DSM 8903} & 70 & Pine wood & ND & ND & 0.67 & [32] \\
\hline & 70 & Sweet sorghum & ND & 1.8 & $1.07^{\mathrm{b}}$ & [33] \\
\hline & 70 & Maize leaves & ND & 1.8 & $0.55^{\mathrm{b}}$ & [33] \\
\hline & 70 & Silphium leaves & ND & 0.5 & $0.29^{\mathrm{b}}$ & [33] \\
\hline & 70 & Sugarcane plant & ND & 2.3 & $0.65^{\mathrm{b}}$ & {$[33]$} \\
\hline $\begin{array}{l}\text { Caldicellulosiruptor bescii } \\
\text { sp. nov. DSM } 6725\end{array}$ & 75 & Switchgrass & Acetate and lactate & ND & 0.16 & [116] \\
\hline
\end{tabular}

a mol $\mathrm{H}_{2} /$ mol pentoses according to the equation: $3 \mathrm{C}_{5} \mathrm{H}_{10} \mathrm{O}_{5}+5 \mathrm{H}_{2} \mathrm{O} \rightarrow 10 \mathrm{H}_{2}+5 \mathrm{CH}_{3} \mathrm{COOH}+5 \mathrm{CO}_{2}$

${ }^{\mathrm{b}} \mathrm{mmol} \mathrm{H}_{2}$ produced/g dry biomass (converted from the reported value) 